\title{
Article \\ On the Geometric Description of Nonlinear Elasticity via an Energy Approach Using Barycentric Coordinates
}

\author{
Odysseas Kosmas *D, Pieter Boom and Andrey P. Jivkov $\mathbb{D}$ \\ Department of MACE, University of Manchester, Oxford Road, Manchester M13 9PL, UK; \\ pieter.boom@manchester.ac.uk (P.B.); andrey.jivkov@manchester.ac.uk (A.P.J.) \\ * Correspondence: odysseas.kosmas@manchester.ac.uk; Tel.: +44-(0)161-306-3727
}

check for updates

Citation: Kosmas, O.; Boom, P.; Jivkov, A.P. On the Geometric Description of Nonlinear Elasticity via an Energy Approach Using Barycentric Coordinates. Mathematics 2021, 9, 1689. https://doi.org/ $10.3390 /$ math 9141689

Academic Editor: Dumitru Baleanu

Received: 5 May 2021

Accepted: 7 July 2021

Published: 19 July 2021

Publisher's Note: MDPI stays neutral with regard to jurisdictional claims in published maps and institutional affiliations.

Copyright: (c) 2021 by the authors. Licensee MDPI, Basel, Switzerland. This article is an open access article distributed under the terms and conditions of the Creative Commons Attribution (CC BY) license (https:// creativecommons.org/licenses/by/ $4.0 /)$.

\begin{abstract}
The deformation of a solid due to changing boundary conditions is described by a deformation gradient in Euclidean space. If the deformation process is reversible (conservative), the work done by the changing boundary conditions is stored as potential (elastic) energy, a function of the deformation gradient invariants. Based on this, in the present work we built a "discrete energy model" that uses maps between nodal positions of a discrete mesh linked with the invariants of the deformation gradient via standard barycentric coordinates. A special derivation is provided for domains tessellated by tetrahedrons, where the energy functionals are constrained by prescribed boundary conditions via Lagrange multipliers. The analysis of these domains is performed via energy minimisation, where the constraints are eliminated via pre-multiplication of the discrete equations by a discrete null-space matrix of the constraint gradients. Numerical examples are provided to verify the accuracy of the proposed technique. The standard barycentric coordinate system in this work is restricted to three-dimensional (3-D) convex polytopes. We show that for an explicit energy expression, applicable also to non-convex polytopes, the general barycentric coordinates constitute fundamental tools. We define, in addition, the discrete energy via a gradient for general polytopes, which is a natural extension of the definition for discrete domains tessellated by tetrahedra. We, finally, prove that the resulting expressions can consistently describe the deformation of solids.
\end{abstract}

Keywords: nonlinear elasticity; general barycentric coordinates; energy minimisation; Lagrange multipliers; null-space method

\section{Introduction and Motivation}

Computational solid mechanics provides approximate solutions for the deformation of continuous domains subjected to changes in boundary conditions [1-6]. The deformation process itself is described by using "intensive quantities" — stresses and strains-and a constitutive relation between them. These quantities have a geometric nature and form continuous tensor fields. The constitutive relation between stresses and strains may vary in the degree of complexity, depending on how the intensive quantities are related to the "extensive quantities" - forces and displacements. For example, the strains can be defined as linearised or nonlinear functions of the displacement gradient, but in all cases they must be symmetric tensors in order to fulfil physical objectivity. On the other hand, stresses can be defined as distributed forces with respect to a specific domain configuration, where the known true or Cauchy stresses are defined with respect to the current/deformed configuration. Furthermore, the simultaneous fulfilment of the balance of linear and angular momenta generates the symmetry of the stress tensor. As a consequence, the differential relations representing the strains as functions of displacements and equilibrium of stresses, together with the constitutive law, form a system of equations, the approximate solution of which is sought either by discretising the underlying solution space or by discretising the operators involved.

For the first approach (discretisation of the solution space), the most prominent example is the well-known finite element method. In this method, the standard finite 
element formulations, which dominate commercial finite element analysis platforms, are based on a limited number of simple element geometries: triangles and quadrilaterals in 2D, and tetrahedra and hexahedra in 3D. While these are sufficient for most practical problems and make the implementation and the solution quite efficient, there are, however, situations where the use of general polyhedra as the indivisible units covering the domain can be really more advantageous. One obvious example of this kind is related to the representation of large polycrystalline microstructures or cellular assemblies, where the need to insert additional discretisation in the polyhedra may lead to computationally very expensive problems. This has led to developments of finite elements of the form of general polyhedra, including those using an arbitrary number of vertices and faces, those using generally non-convex polyhedra, and those using polyhedra with nonplanar faces [7-9]. In parallel, such general polyhedra proved to be the driving force for the recent development of the virtual element method $[10,11]$. However, to the best of our knowledge, methods that use general polyhedral meshing tools and then employ the subsequent solvers have not become fully established to date, and they remain in the academic domain.

The second approach (discretisation of the operators) is, to a large extent, based on the discrete differential geometry and was kept under development during the last 20 years. In this method, the discrete structure of the analysed solid at a given length-scale is considered as a starting point, i.e., the discretised computational domain is defined via the finite discrete nature of the solid constitution, and can be seen as an assembly of cells of arbitrary sizes and shapes. Concrete examples of this approach put further effort in order to preserve key properties of the system in terms of important invariants, such as energy, by proper discretisation of the operators. Even though these schemes have been well figured out/formulated and tested for a wide range of physical problems involving scalar fields, only a few of them have been proven to be stable when solving solid mechanics problems involving vector fields [12-14]. A notable approximation within this approach is the representation of the discrete system with a graph (contour) that allows rather simple formulations for the elasticity [15], the elasto-plasticity [16] and the elasto-plasticity involving damage [17].

For both of the aforementioned approaches in computational solid mechanics, the mesh quality plays crucial role in numerical simulations. For example, in several methods that rely on Voronoi meshes there may appear spurious solutions, mainly due to different scales of edges and faces (presence of small edges and/or faces), see, e.g., in [18] and references therein. Similar problems arising from mesh quality are present in several other methods, see, for example, in [13]. A common approach utilised to overcome such difficulties is the application of re-meshing: an initial tetrahedral mesh of any quality is used to create a new one with improved quality by appropriate merging of neighbouring tetrahedra. Examples of this technique can be found, e.g., in [19], using mesh-free methods, and in [20] using discontinuous Galerkin methods. Such a treatment, however, is not applicable in situations where a mesh representing some physically-based structure is required, e.g., an assembly of polytopes, and possible large differences in scales need to be handled. The effect of scale differences is quite strong due to the representation of differential operators in either approach, and could be overcome by an energy-based formulation.

The main aim of this work, is the consideration of the above open questions, focusing on the derivation of an appropriate energy-based model within the field of computational solid mechanics. This model would combine a continuum geometric description for the stresses and strains in the context of nonlinear elasticity, and a discrete energy formulation for tetrahedra as well as arbitrary convex polyhedra. The paper is structured as follows. We first recall the geometric description of deformation and the continuous definition of elastic (stored, conservative) energy in Section 2. This is subsequently used to derive a discrete energy representation in tetrahedral elements through the use of standard barycentric coordinates, Section 3. The problem of elastic deformation is formulated as an "energy minimisation problem", where the boundary conditions are imposed via Lagrange 
multipliers. The null-space method is then employed to eliminate the Lagrange multipliers, Section 4 . The formulation of ... and the solution procedure are validated in Section 5 by comparison with analytical solutions for two examples: a cantilever beam subjected to uniformly distributed load, in Section 5.1, and a domain with a spherical hole subjected to remote tension, in Section 5.2. Finally, an energy model for general polytopes is proposed in Section 6. This is achieved by extending the definition of the standard barycentric coordinates to such polytopes, deriving weighted ones, and proving that these can define an energy functional that fully describes the physical system.

\section{Deformation and Energy of Conservative Solids}

We will first review some of the basic definitions of nonlinear elasticity from a geometric perspective. We start by identifying a material body with a (smooth) Riemannian manifold $B$ and consider a time-dependent deformation of this body to the ambient space Riemannian manifold $S$ described by [1]

$$
\varphi_{t}: B \rightarrow \varphi_{t}(B),
$$

or, for simplicity,

$$
\varphi: B \rightarrow S,
$$

see Figure 1. The points within the body are given with their coordinates with respect to a global coordinate system: $\mathbb{X}=\left[\begin{array}{lll}X_{1} & X_{2} & X_{3}\end{array}\right]^{T}$-in the initial (reference) configuration, and $\mathbb{x}=\left[\begin{array}{lll}x_{1} & x_{2} & x_{3}\end{array}\right]^{T}$-in the current (deformed) configuration. For these we can rewrite the deformation map (2) as

$$
\mathbb{x}=\varphi(\mathbb{X}) \equiv \mathbb{x}(\mathbb{X}) .
$$

The map $\varphi$ is considered to be a diffeomorphism, i.e., an invertible differentiable map with differentiable inverse. Manifolds $B$ and $S$ are considered to be equipped with a metric tensor field $G$. This positive definite second-order tensor field is expressed by symmetric tensors in the tangent space at points of the manifold, denoted by $T_{\mathbb{X}} B$ on $B$ and $T_{\mathbb{X}} S$ on $S$.

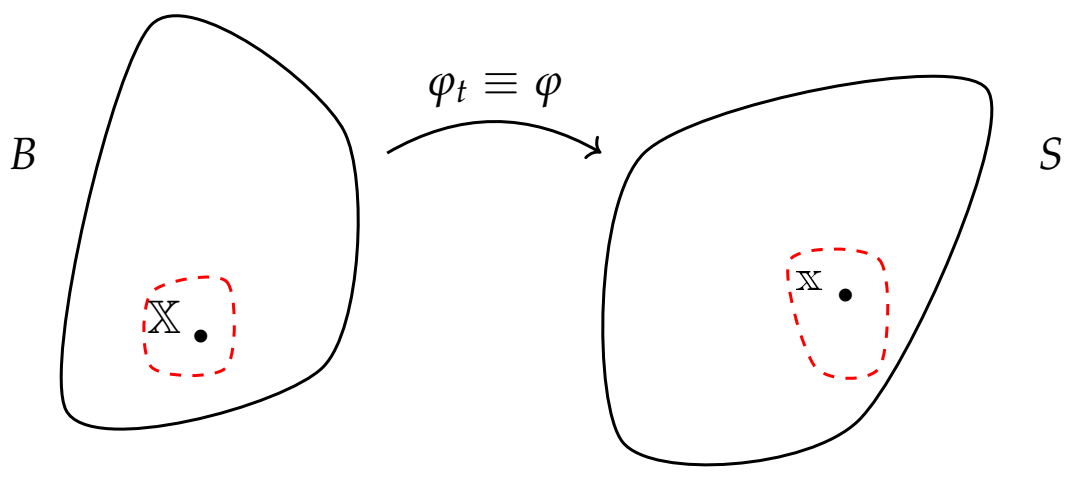

Figure 1. Initial $B$ and current $S$ configurations of a domain related by map $\varphi_{t} \equiv \varphi$.

A deformation (3) can also be represented by the deformation gradient $\mathcal{F}$, which is the tangent map of $\varphi$, i.e., the map between the tangent space on $B$ and the tangent space on $S[1,2]$ :

$$
\mathcal{F}(\mathbb{X}): T_{\mathbb{X}} B \rightarrow T_{\mathbb{X}} S .
$$

In the local coordinate charts for $\mathbb{X}$ and $\mathbb{x}$, the components $\mathcal{F}_{i j}$ of $\mathcal{F}$ are given by

$$
\mathcal{F}_{i j}=\frac{\partial x_{i}}{\partial X_{j}}=x_{i, j} \quad \text { for } \quad i, j=1,2,3 .
$$

The deformation gradient is a non-singular two-point tensor, i.e., maps the tangent spaces of the two configurations, with a positive determinant, denoted by J. The determinant measures the ratio between the current and initial infinitesimal volume at a given 
material point, and we note that the condition $\mathbb{J}=1$ represents a volume preserving deformation. $\mathcal{F}$ can be multiplicatively decomposed in two possible ways:

$$
\mathcal{F}=\mathcal{V} \mathcal{R}=\mathcal{R} U,
$$

where $\mathcal{R}$ is a proper orthogonal rotation tensor (considering that body and space are of the same Riemannian manifold), and $\mathcal{V}$ and $\mathcal{l}$ are the so-called left (spatial, current) and right (material, reference) stretch tensors, respectively, which are symmetric positive-definite as $\mathbb{J}>0$. This reflects the two possible representations of the motion: (1) first rotation of a reference unit triad to a current unit triad, followed by its stretching in the current configuration, and (2) stretching of a reference unit triad, followed by rotation to a current triad. The two stretch tensors have identical three positive eigenvalues $-\lambda_{1}, \lambda_{2}$, and $\lambda_{3}$-representing the principal stretches of the deformation.

In this work, we will consider solids undergoing conservative deformation, i.e., where the solid stores no energy on a complete reversal of the deformation [5]. This behaviour covers the cases of linear and nonlinear elasticity, where all work done on the system by changing boundary conditions from one (initial) to another (current) configuration is stored as an elastic energy, which is exactly equal to the energy required to restore the initial configuration by reversed change of boundary conditions.

An elastic energy formulation in terms of deformation must be invariant with respect to rigid body rotations $[1,2,4]$, thus existing formulations are based on the stretch tensors or some functions of their invariants. We will use one such formulation, which is based on invariants of the so-called left Cauchy-Green strain tensor $\mathcal{B}$, given by the map $\mathcal{B}(\mathbb{x})$ : $T_{\mathbb{X}} S \rightarrow T_{\mathbb{X}} S$ or by

$$
\mathcal{B}=\mathcal{V}^{2} \equiv \mathcal{F F}^{T},
$$

where $\mathcal{F}^{T}$ maps covectors in the cotangent bundle of $S$ (or $T^{*} S$ ) to covectors in the cotangent bundle of $B\left(\right.$ or $\left.T^{*} B\right)$, see also in [21-25]. It can be easily shown that $\mathcal{B}$ is objective, i.e., frame-independent, tensor.

One set of invariants of $\mathcal{B}$ is given by $[1,2,4]$

$$
\mathbb{I}_{1}=\operatorname{tr}\left(\mathcal{F F}^{T}\right), \mathbb{I}_{2}=\frac{1}{2}\left[\left(\operatorname{tr}\left(\mathcal{F F}^{T}\right)\right)^{2}-\left(\operatorname{tr}\left(\mathcal{F F}^{T}\right)^{2}\right)\right], \mathbb{I}_{3}=\operatorname{det}\left(\mathcal{F F}^{T}\right)=\mathbb{J}^{2}
$$

which can be written in terms of the three principal stretches as

$$
\mathbb{I}_{1}=\lambda_{1}^{2}+\lambda_{2}^{2}+\lambda_{3}^{2}, \quad \mathbb{I}_{2}=\left(\lambda_{1} \lambda_{2}\right)^{2}+\left(\lambda_{1} \lambda_{3}\right)^{2}+\left(\lambda_{2} \lambda_{3}\right)^{2}, \quad \mathbb{I}_{3}=\lambda_{1} \lambda_{2} \lambda_{3},
$$

Another set of invariants, used to define a large class of non-linear elastic behaviours, is derived from (9) as

$$
\overline{\mathbb{I}}_{1}=\frac{\mathbb{I}_{1}}{\mathbb{J}^{2 / 3}}, \quad \overline{\mathbb{I}}_{2}=\frac{\mathbb{I}_{2}}{\mathbb{J}^{4 / 3}}, \quad \overline{\mathbb{I}}_{3}=\sqrt{\operatorname{det}\left(\mathcal{F F}^{T}\right)}=\mathbb{J} .
$$

The elastic energy density of a simple generalised Neo-Hookean material is defined in terms of the second set by $[2,4]$

$$
\mathcal{H}=\frac{\mu}{2}\left(\overline{\mathbb{I}}_{1}-3\right)+\frac{\kappa}{2}(\mathbb{J}-1)^{2},
$$

where $\mu$ and $\kappa$ are material-dependent parameters, which in the small strain approximation are known as shear and bulk moduli, respectively.

The integral of the energy density over the solid domain gives the total elastic (stored, potential) energy, which by the principle of stationary action must be minimum for the true deformation; derivation is shown in Appendix A. This can be understood as the system storing the minimal amount of energy for the change of boundary conditions between the initial and the current configuration, or as the change of boundary conditions doing the 
minimal amount of work (which is equal to the stored energy) to deform the solid from the initial to the current configuration.

While the solid deformation will be formulated as an elastic energy minimisation problem in this work, using the energy density expression (11), the stress tensor will be required for comparisons with known solutions of test problems. The (true) Cauchy stress tensor, $\sigma$, is the derivative of the elastic energy density function with respect to $\mathcal{B}$, which for the case of Neo-Hookean materials can be found as

$$
\sigma_{i j}=\frac{\mu}{\mathbb{J}^{5 / 3}}\left(\mathcal{B}_{i j}-\frac{1}{3} \mathcal{B}_{k k} \delta_{i j}\right)+\kappa(\mathbb{J}-1) \delta_{i j},
$$

where $\delta_{i j}$ is the Kroneckers delta.

Interesting relations between the material parameters and energy can be established via the derivatives of the energy function with respect to the invariants of $\mathcal{F}$; these are shown in the Appendix $C$ where the formulation is specialised to linear elasticity, i.e., infinitesimal deformation approximation.

\section{Discrete Energy of Tetrahedral Cells}

First, we consider a subdivision of the material manifold $B$ into tetrahedra. For a given tetrahedron in $\mathbb{R}^{3}$ with vertices $\mathbb{X}^{a}, \mathbb{X}^{b}, \mathbb{X}^{c}$ and $\mathbb{X}^{d}$, any point $\mathbb{X}$ induces a partition described via

$$
\mathbb{X}=\gamma^{a} \mathbb{X}^{a}+\gamma^{b} \mathbb{X}^{b}+\gamma^{c} \mathbb{X}^{c}+\gamma^{d} \mathbb{X}^{d}
$$

where $\gamma^{a}, \gamma^{b}, \gamma^{c}, \gamma^{d} \in \mathbb{R}$ are generalised barycentric coordinates. These are the ratios of the partitioned signed volumes $V_{i}$ and the tetrahedral volume $\left(V^{t}\right)$, see Figure 2 . We introduce the vector of barycentric coordinates as

$$
\mathbb{\Gamma}=\left[\begin{array}{llll}
\gamma^{a} & \gamma^{b} & \gamma^{c} & \gamma^{d}
\end{array}\right]^{T}
$$

Further, we define a $4 \times 4$ matrix $\mathcal{S}^{r}$ describing the tetrahedron shape in the reference configuration by

$$
\mathcal{S}^{r}=\left[\begin{array}{llll}
\tilde{\mathbb{X}}^{a} & \tilde{\mathbb{X}}^{b} & \tilde{\mathbb{X}}^{c} & \tilde{\mathbb{X}}^{d}
\end{array}\right],
$$

where each column contains corresponding nodal coordinates in the reference system with an additional element " 1 ", e.g., for the first node we have

$$
\tilde{\mathbb{X}}^{a}=\left[\begin{array}{llll}
X_{1}^{a} & X_{2}^{a} & X_{3}^{a} & 1
\end{array}\right]^{T}
$$

and similarly for the remaining three nodes (upper indexes here indicate vertices, see Figure 3 , and lower the $i$-th component of it, $i=1,2,3)$. Using this, any point $\mathbb{X}$ in the reference configuration can be expressed by

$$
\tilde{\mathbb{X}}=\mathcal{S}^{r} \mathbb{\Gamma} .
$$

Similarly we define a $4 \times 4$ matrix $\mathcal{S}^{d}$ describing the tetrahedron shape in the deformed configuration by

$$
S^{d}=\left[\begin{array}{llll}
\tilde{\mathbb{X}}^{a} & \tilde{\mathbb{X}}^{b} & \tilde{\mathbb{X}}^{c} & \tilde{\mathbb{X}}^{d}
\end{array}\right] .
$$

Using this, any point $\mathrm{x}$ in the deformed configuration can be expressed by

$$
\tilde{\mathbf{x}}=\mathcal{S}^{d} \mathbb{\Gamma} .
$$

These expressions enable us to define the map between the reference and current configuration for any tetrahedral cell (see Figure 3) by

$$
\tilde{\mathbb{X}}=S^{d}\left(S^{r}\right)^{-1} \tilde{\mathbb{X}}
$$



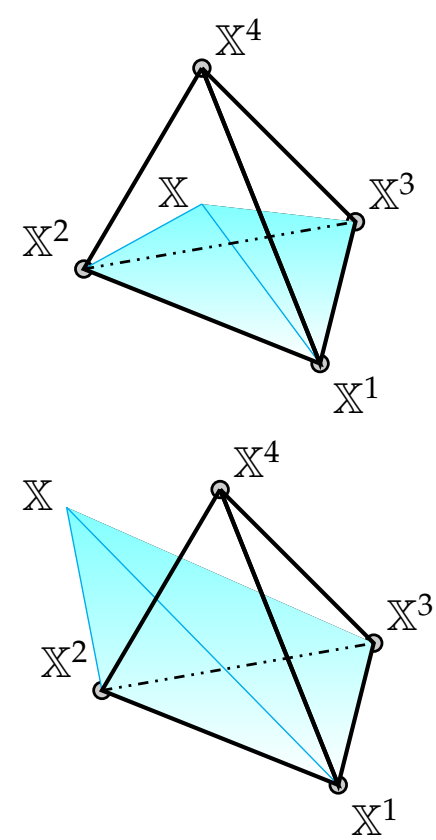
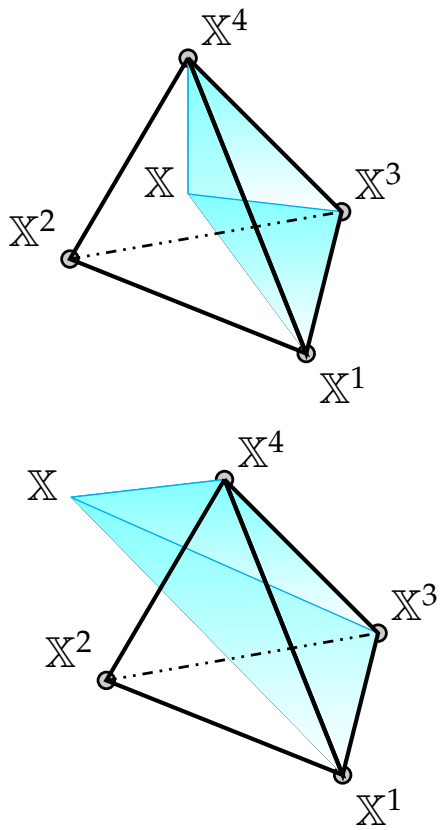

Figure 2. Examples of partitioned volumes $V_{I}$ associated with point $\mathbb{X}$ : (top) two figures illustrate two volumes for point inside the tetrahedron; (bottom) two figures illustrate two volumes for a point outside the tetrahedron.
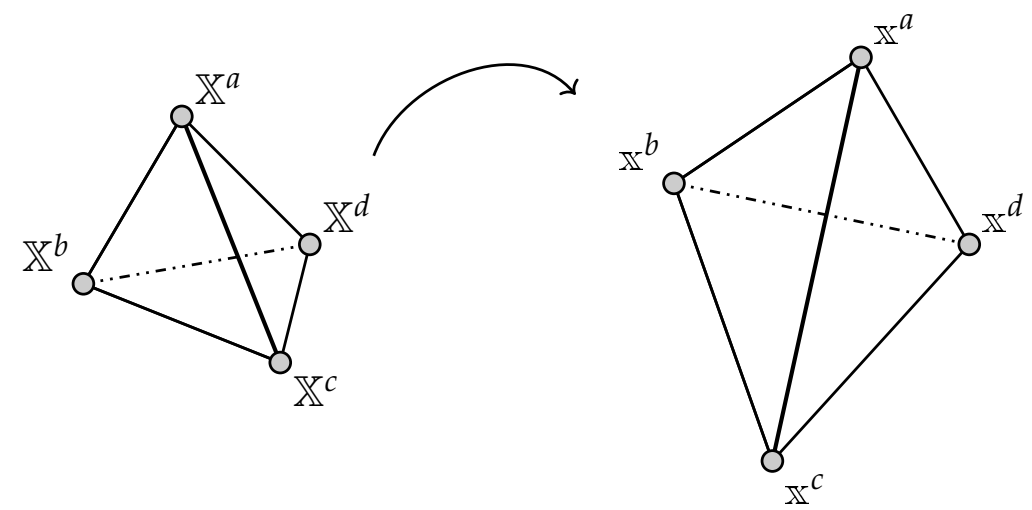

Figure 3. The deformation of a $3 \mathrm{D}$ tetrahedral element.

From another side, the physical map between the reference and the current configuration of a tetrahedron can be given by

$$
\mathbb{x}=\mathcal{F} \mathbb{X}+\mathbb{t}
$$

where $t=\left[t_{i}\right], i=1,2,3$ are the components of a translation vector, and $\mathcal{F}$ is the deformation gradient which is assumed to have positive determinant.

After some algebra, it can be shown that $S^{d}\left(\mathcal{S}^{r}\right)^{-1}$ is the following a $4 \times 4$ block matrix:

$$
\mathcal{S}^{d}\left(\mathcal{S}^{r}\right)^{-1}=\left[\begin{array}{r|r}
\mathcal{F} & \mathbb{t} \\
\hline \mathbb{O}^{T} & 1
\end{array}\right],
$$

where $\mathbb{O}$ is a zero vector of size three.

With the knowledge of $\mathcal{F}$, the discrete energy of a tetrahedron is calculated by Equation (11), using the invariants given in Equation (10). The sum of energies of all tetrahedrons defines the discrete energy functional of the system. 


\section{Lagrange Multipliers Using Null-Space Method}

When using the discrete energy functional proposed in Section 3 the Euler-Lagrange Equation (A4) are incomplete, and need to be complemented by prescribing Dirichlet (essential) and Neumann (natural) boundary conditions, see Appendix B. For the proposed formulation via energy minimisation, direct application of the boundary conditions is challenging and in such cases Lagrange multipliers have been extensively used [26]. This has been motivated by the fact that both essential and natural boundary conditions can be expressed as constraints and thus enforced by Lagrange multipliers, resulting in the following constrained Euler-Lagrange equations:

$$
\frac{d}{d X_{j}} \frac{\partial \mathcal{H}}{\partial\left(\partial x_{i} / \partial X_{j}\right)}-\frac{\partial \mathcal{H}}{\partial x_{i}}-\left[C\left(X_{j}\right)\right]^{T} \lambda^{j}=0,
$$

for $i, j=1,2,3$, where $C\left(X_{j}\right)$ are the constraints and $\lambda^{j}$ are the Lagrange multipliers.

The introduction of Lagrange multipliers increases the number of unknowns, and in order to reduce them to the number of unknown displacements in the system we use the discrete null-space method of [26], which eliminates all Lagrange multipliers. For this, we define a null-space matrix $N\left(X_{j}\right)$, which satisfies

$$
C\left(X_{j}\right) N\left(X_{j}\right)=0 .
$$

Multiplying from the left with its transpose, Equation (23) becomes

$$
\left[N\left(X_{j}\right)\right]^{T}\left(\frac{d}{d X_{j}} \frac{\partial \mathcal{H}}{\partial\left(\partial x_{i} / \partial X_{j}\right)}-\frac{\partial \mathcal{H}}{\partial x_{i}}\right)=0 .
$$

This leads to a number of equations equal to the number of unknown degrees of freedom. Importantly, this technique has been proven to be energy consistent, meaning that energy is neither dissipated nor gained artificially during the numerical process [26-31].

\section{Numerical Examples}

A canonical way to test a proposal for numerical solution of boundary value problems in elasticity is to examine the solution behaviour for several simple deformation modes: volumetric expansion, pure shear, and possibly unconstrained uniaxial extension/compression. While we have tested these modes successfully, the inclusion of the results would not be of significant value to this work. Instead, we provide results for two known elasticity problems, where the combined effect of the different deformation modes is tested: a cantilever beam subjected to a uniformly distributed load and a cube with a spherical hole subjected to tension. We will present and compare results for displacements in the cantilever case, and for stresses in the cube case.

\subsection{Cantilever Beam Subjected to a Regular Distributed Load}

First, we consider the deformation of a three-dimensional cantilever beam subjected to a uniformly distributed load [4]. The beam has dimensions $10 \times 2 \times 2$ and is discretised into 5802 tetrahedral elements using 1322 nodes, see Figure 4 . The material of the beam has properties $E=3 \times 10^{7} \mathrm{kPa}$ and $v=0.3$.

The uniformly distributed load is applied in ten increments with step $f_{i}=4 \mathrm{kN} / \mathrm{cm}^{2}$, and the solution is compared with linear and geometrically nonlinear finite elements analyses performed with identical tetrahedral elements. The comparison shown in Figure 5 illustrates that the calculated deflection is in excellent agreement with the geometrically nonlinear finite element solution. This is the first demonstration that the proposed method based on the minimisation of energy obtained by the barycentric map. 


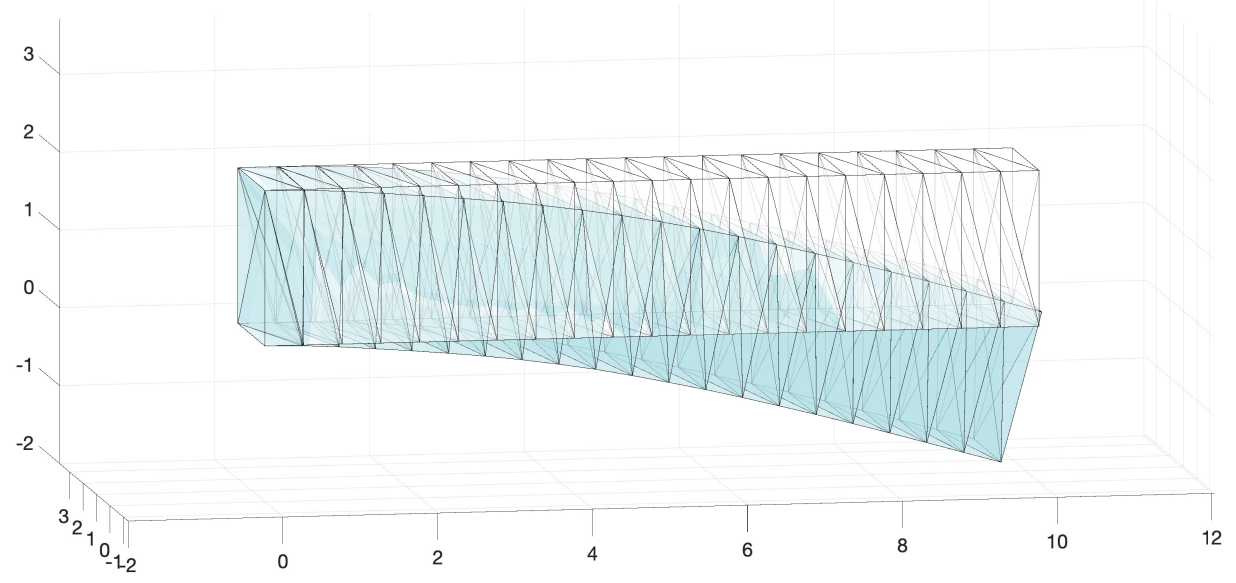

Figure 4. 3d cantilever beam.

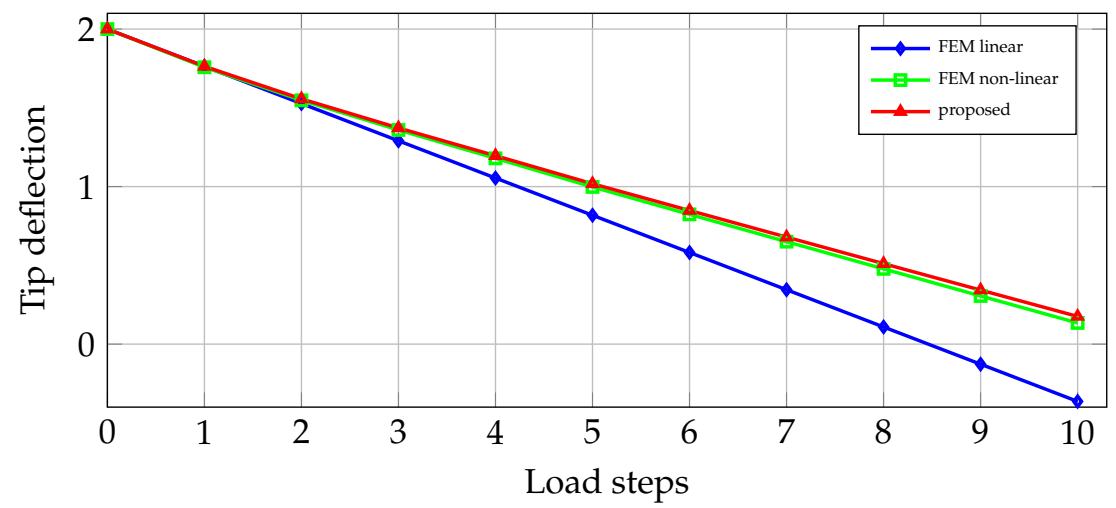

Figure 5. The deflection of the geometrically nonlinear case of a cantilever beam subjected to a regular distributed load calculated using finite element method with tetrahedral elements (FEM-tet) and the proposed scheme.

\subsection{Cube with a Spherical Hole}

Second, we consider the deformation of a cube with a spherical hole subjected to tensile load (Figure 6). The cube dimensions are $20 \times 20 \times 20$, and the spherical cavity has a radius $r=1$ and centre at the cube centre. Due to symmetries, only one-eight of the cube is considered and tessellated with approximately 100 tetraherdrons. The material properties are the same as in the cantilever example. Uniform tensile load parallel to the $x$ axis is applied.

The problem of a continuum domain with a spherical hole subjected to remote stress has an analytical solution [32]. Specifically the normal stress to the $x, y$ plane $(z=0)$ is

$$
\sigma_{33}=\frac{4-5 v}{2(7-5 v)}\left(\frac{r}{x}\right)^{3}+\frac{9}{2(7-5 v)}\left(\frac{r}{x}\right)^{5}+1 .
$$

This analytical solution is compared with the results obtained with the proposed method in Figure 7: analytical solution is plotted with blue line, calculated stresses are plotted with red line. The demonstrated good agreement between the two lends further support to the proposed approach. 


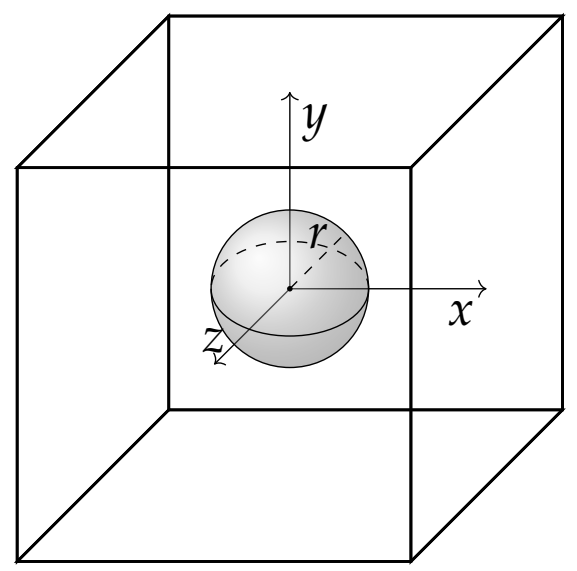

Figure 6. Cube with a spherical hole.

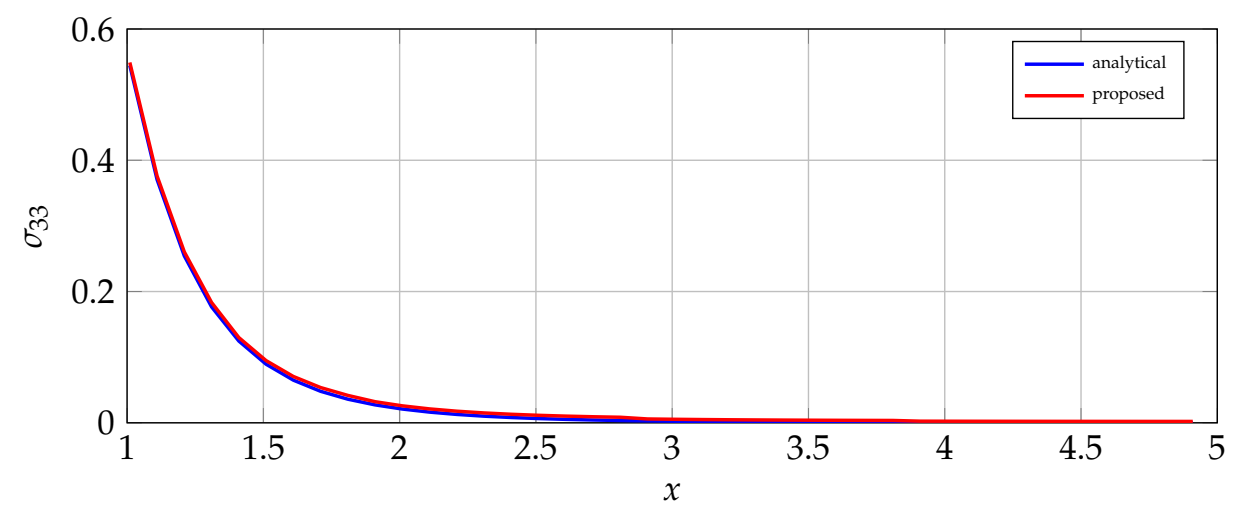

Figure 7. Stress distribution around the hole for distance $x>1$ from the centre of the hole (size does not corresponds to example).

The approach can be tested further against various analytical solutions, but the implementation requires additional work to reduce the computational cost. Additionally, more work must be done on the mesh quality, in order to demonstrate how the number, size and shape of the tetrahedra matter in the calculations. One part of an ongoing work is an implementation of a parallel solver that will massively reduce that time.

\section{Discrete Energy on General Polytopes Using Weighted Barycentric Coordinates}

In order to define a discrete energy for general three-dimensional elements, we will follow an approach similar to the one described in Section 3. The extension requires first to define general/weighted barycentric coordinates on general polytopes and then to use them to express points of the material domain with respect to the tessellation nodes.

\subsection{Standard Barycentric Coordinates Revisited}

We start with rewriting the standard barycentric coordinates in a form suitable for generalisation. With respect to a convex polytope with vertices $\mathbb{X}_{I}$ for $I=1, \ldots, n$ (where $n \geq 4$ ), any point $\mathbb{X} \in \mathbb{R}^{3}$ can written as [33]

$$
\mathbb{X}=\sum_{I=1}^{n} \gamma_{I} \mathbb{X}_{I}, \quad \sum_{I=1}^{n} \gamma_{I}=1,
$$

where $\gamma_{I}$ are the generalised barycentric coordinates of $\mathbb{X}$. This can be also written in the form

$$
\sum_{I=1}^{n} w_{I}\left(\mathbb{X}_{I}-\mathbb{X}\right)=0, \quad \sum_{I=1}^{n} w_{I} \neq 0, \quad \text { with } \quad \gamma_{I}=\frac{w_{I}}{\sum_{I=1}^{n} w_{I}}
$$


where the weight functions $w_{I}$ can be appropriately defined. In [33] for example, the authors define the weight functions via the partition volumes

$$
V_{I}=\left\{\mathbb{X}_{1}, \mathbb{X}_{2}, \ldots, \mathbb{X}_{I-1}, \mathbb{X}, \mathbb{X}_{I+1}, \ldots, \mathbb{X}_{n}\right\}_{\text {volume }}
$$

and the positive functions $c_{I}>0$, so that

$$
w_{I}=\frac{c_{I+1} V_{I+1}+c_{I} V_{I}+c_{I-1} V_{I-1}}{V_{I+1} V_{I-1}} .
$$

The sum of the weight functions becomes

$$
W=\sum_{I=1}^{n} w_{I}=\sum_{I=1}^{n} \frac{c_{I} V}{V_{I+1} V_{I-1}},
$$

where $V$ is the volume of the polytope.

The requirement for $c_{I}$ is that these are arbitrary positive functions. A rather general definition has been proposed in [33]:

$$
c_{I}=\left|\mathbb{X}_{I}-\mathbb{X}\right|^{\alpha} .
$$

Notably, the selection $\alpha=0$ results in the known Wachspress coordinates, while the selection $\alpha=1$ provides the mean value coordinates.

\subsection{Weighted Barycentric Coordinates on General Polytopes}

We will now propose an extended version of barycentric coordinates on general polytopes that will be used to formulate an expression of the internal energy. To do so, we use the signed partitioned volumes given by Equation (29) and extend the definition of weight functions given by Equation (31).

Extending the barycentric coordinate expressions to general polytopes is challenging, because one needs to address issues arising from the definition of the weight functions in Equation (31). The problems stem mainly from the denominator, the product of the volumes $V_{I-1}$ and $V_{I+1}$ (or areas in two dimensions). For non-convex polytopes, this product might become negative. In the following, we restrict ourselves to arbitrary (nonplatonic) tetrahedral elements, but the generalisation to any general polytope follows clearly. To bypass the possibility of negative denominator, we define the volume using cross-products in the following way:

$$
V=\frac{1}{6}\left|\left(\mathbb{X}_{1}-\mathbb{X}_{2}\right) \times\left(\mathbb{X}_{1}-\mathbb{X}_{3}\right)\right|\left|\mathfrak{h}_{\mathbb{X}_{4},\left(\mathbb{X}_{1}, \mathbb{X}_{2}, \mathbb{X}_{3}\right)}\right|,
$$

where $\mathbb{h}_{\mathbb{X}_{I},\left(\mathbb{X}_{1}, \mathbb{X}_{2}, \mathbb{X}_{3}\right)}$ is the vector normal to the triangle face forming from points $\left(\mathbb{X}_{1}, \mathbb{X}_{2}, \mathbb{X}_{3}\right)$ to the point $\mathbb{X}_{I}$.

We can now rewrite the weights of (31) using the proposed volume definition to obtain

$$
\begin{aligned}
& W=\sum_{I=1}^{n} \frac{6^{2} c_{I} V}{\left.\left|\left(\mathbb{X}_{1}-\mathbb{X}_{2}\right) \times\left(\mathbb{X}_{1}-\mathbb{X}_{3}\right)\right|\left|\mathfrak{h}_{\mathbb{X},\left(\mathbb{X}_{1}, \mathbb{X}_{2}, \mathbb{X}_{3}\right)}\right|\left|\left(\mathbb{X}_{1}-\mathbb{X}_{3}\right) \times\left(\mathbb{X}_{1}-\mathbb{X}_{4}\right)\right| \mid \mathfrak{h}_{\mathbb{X},\left(\mathbb{X}_{1}, \mathbb{X}_{3}, \mathbb{X}_{4}\right)}\right)} \\
& =\sum_{I=1}^{n} \frac{6 c_{I}\left|\mathbb{h}_{\mathbb{X}_{4},\left(\mathbb{X}_{1}, \mathbb{X}_{2}, \mathbb{X}_{3}\right)}\right|}{\left|\left(\mathbb{X}_{1}-\mathbb{X}_{3}\right) \times\left(\mathbb{X}_{1}-\mathbb{X}_{4}\right)\right|\left|\mathfrak{h}_{\mathbb{X},\left(\mathbb{X}_{1}, \mathbb{X}_{2}, \mathbb{X}_{3}\right)}\right|\left|\mathfrak{h}_{\mathbb{X},\left(\mathbb{X}_{1}, \mathbb{X}_{3}, \mathbb{X}_{4}\right)}\right|} \\
& =\sum_{I=1}^{n} \frac{6 c_{I}\left|\mathbb{h}_{\mathbb{X}_{4},\left(\mathbb{X}_{1}, \mathbb{X}_{2}, \mathbb{X}_{3}\right)}\right|}{\left.\left|\mathbb{X}_{1}-\mathbb{X}_{3}\right|\left|\mathbb{X}_{1}-\mathbb{X}_{4}\right| \mid \mathfrak{h}_{\mathbb{X},\left(\mathbb{X}_{1}, \mathbb{X}_{2}, \mathbb{X}_{3}\right)}\right)\left|\mathbb{h}_{\mathbb{X},\left(\mathbb{X}_{1}, \mathbb{X}_{3}, \mathbb{X}_{4}\right)}\right| \sin \left(\left(\mathbb{X}_{1}-\mathbb{X}_{3}\right),\left(\mathbb{X}_{1}-\mathbb{X}_{4}\right)\right)},
\end{aligned}
$$


With some functions $d_{I}>0$ this can be written as

$$
\begin{aligned}
W & =\sum_{I=1}^{n} \frac{d_{I}}{\left|\mathbb{X}_{1}-\mathbb{X}_{3}\right|\left|\mathbb{X}_{1}-\mathbb{X}_{4}\right| \sin \left(\left(\mathbb{X}_{1}-\mathbb{X}_{3}\right),\left(\mathbb{X}_{1}-\mathbb{X}_{4}\right)\right)} \\
& =\sum_{I=1}^{n} d_{I} \frac{\cot \left(\left(\mathbb{X}_{1}-\mathbb{X}_{3}\right),\left(\mathbb{X}_{1}-\mathbb{X}_{4}\right)\right)}{\left(\mathbb{X}_{1}-\mathbb{X}_{3}\right) \cdot\left(\mathbb{X}_{1}-\mathbb{X}_{4}\right)}
\end{aligned}
$$

As long as the dot product in the denominator is not zero, the barycentric coordinates that use the proposed weight functions are well defined for both convex and non-convex polytopes. Furthermore, the standard barycentric coordinates can be recovered from this definition.

\subsection{Energy from Angles and Lengths}

In order to understand more the angles introduced in (35), we will relate them to appropriate lengths of edges. For illustration, we will restrict ourselves to two dimensions, although the extension to three dimensions follows a similar path. We consider the triangle formed by points with coordinates $\mathbb{X}_{a}, \mathbb{X}_{b}$ and $\mathbb{X}_{c}$ (Figure 8 ). The three angles $\theta_{a}, \theta_{b}$ and $\theta_{c}$ are opposite to the edges of lengths $\left|\mathbb{h}_{\mathbb{X}_{b}, \mathbb{X}_{c}}\right|,\left|\mathbb{h}_{\mathbb{X}_{a}, \mathbb{X}_{c}}\right|$ and $\left|\mathfrak{h}_{\mathbb{X}_{a}, \mathbb{X}_{b}}\right|$, respectively.

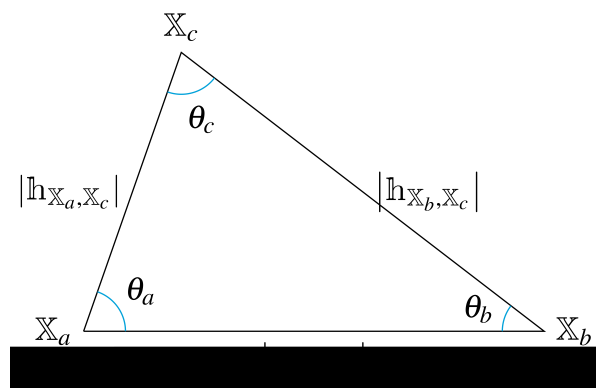

Figure 8. Two-dimensional triangle formed from points with nodal positions $\mathbb{X}_{a}, \mathbb{X}_{b}$ and $\mathbb{X}_{c}$.

Considering each angle to be a function of edge lengths, we can write the following expressions:

$$
\cos \left(\theta_{a}\right)=\frac{\left|\mathfrak{h}_{\mathbb{X}_{a}, \mathbb{X}_{c}}\right|^{2}+\left|\mathbb{h}_{\mathbb{X}_{a}, \mathbb{X}_{b}}\right|^{2}-\left|\mathbb{h}_{\mathbb{X}_{b}, \mathbb{X}_{c}}\right|^{2}}{2\left|\mathbb{h}_{\mathbb{X}_{a}, \mathbb{X}_{c}}\right|\left|\mathbb{h}_{\mathbb{X}_{a}, \mathbb{X}_{b}}\right|} .
$$

By taking the derivative with respect to $\left|\mathbb{h}_{\mathbb{X}_{b}, \mathbb{X}_{c}}\right|$, we have

$$
\frac{\partial \theta_{a}}{\partial\left|\mathfrak{h}_{\mathbb{X}_{b}, \mathbb{X}_{c}}\right|}=\frac{\left|\mathfrak{h}_{\mathbb{X}_{b}, \mathbb{X}_{c}}\right|}{\left|\mathfrak{h}_{\mathbb{X}_{a}, \mathbb{X}_{c}}\right|\left|\mathbb{h}_{\mathbb{X}_{a}, \mathbb{X}_{b}}\right| \sin \left(\theta_{a}\right)},
$$

which, writing the area of the triangle as

$$
A=\frac{1}{2}\left|\mathfrak{h}_{\mathbb{X}_{a}, \mathbb{X}_{c}}\right|\left|\mathfrak{h}_{\mathbb{X}_{a}, \mathbb{X}_{b}}\right| \sin \left(\theta_{a}\right)
$$

results in

$$
\frac{\partial \theta_{a}}{\partial\left|\mathbb{h}_{\mathbb{X}_{b}, \mathbb{X}_{c}}\right|}=\frac{\left|\mathbb{h}_{\mathbb{X}_{b}, \mathbb{X}_{c}}\right|}{2 A}
$$

Similarly, we can calculate the derivative of (36) with respect to $\left|\mathbb{h}_{\mathbb{X}_{a}, \mathbb{X}_{c}}\right|$ as

$$
\frac{\partial \theta_{a}}{\partial\left|\mathbb{h}_{\mathbb{X}_{a}, \mathbb{X}_{c}}\right|}=-\frac{\left|\mathfrak{h}_{\mathbb{X}_{b}, \mathbb{X}_{c}}\right| \cos \left(\theta_{c}\right)}{2 A}
$$


Finally, to connect the cotangent of an angle, required in (35), we define

$$
\xi_{a}=\frac{1}{2}\left|\mathbb{h}_{\mathbb{X}_{b}, \mathbb{X}_{c}}\right|^{2}, \quad \xi_{b}=\frac{1}{2}\left|\mathbb{h}_{\mathbb{X}_{a}, \mathbb{X}_{c}}\right|^{2}, \quad \xi_{c}=\frac{1}{2}\left|\mathfrak{h}_{\mathbb{X}_{a}, \mathbb{X}_{b}}\right|^{2}
$$

and observe that

$$
\frac{\partial \cot \left(\theta_{a}\right)}{\partial \xi_{b}}=\frac{1}{\left|\mathfrak{h}_{\mathbb{X}_{a}, \mathbb{X}_{c}}\right|} \frac{\partial \cot \left(\theta_{a}\right)}{\partial\left|\mathfrak{h}_{\mathbb{X}_{a}, \mathbb{X}_{c}}\right|} .
$$

The last expression, combined with (40), provides

$$
\frac{\partial \cot \left(\theta_{a}\right)}{\partial \xi_{b}}=\frac{\left|\mathbb{h}_{\mathbb{X}_{b}, \mathbb{X}_{c}}\right| \cos \left(\theta_{c}\right)}{2 \sin ^{2}\left(\theta_{a}\right)\left|\mathfrak{h}_{\mathbb{X}_{a}, \mathbb{X}_{c}}\right|} .
$$

This result gives us a perspective to understand the weights defined by (35) via the derivatives with respect to edge lengths. In addition, due to symmetries we can obtain

$$
\frac{\partial \cot \left(\theta_{b}\right)}{\partial \xi_{a}}=\frac{\left|\mathfrak{h}_{\mathbb{X}_{b}, \mathbb{X}_{c}}\right| \cos \left(\theta_{c}\right)}{2 \sin ^{2}\left(\theta_{a}\right)\left|\mathbb{h}_{\mathbb{X}_{a}, \mathbb{X}_{c}}\right|}
$$

and thus

$$
\frac{\partial \cot \left(\theta_{a}\right)}{\partial \xi_{b}}=\frac{\partial \cot \left(\theta_{b}\right)}{\partial \xi_{a}} .
$$

The expressions relating cotangent of angles with lengths can be used to formulate an energy expression.

Total energy given by (A2) can be calculated using the energy of any triangle element, i.e., (A1) as

$$
\kappa_{i} \simeq \int_{\xi_{a, \zeta_{b}, \xi_{c}}} \sum_{I=1}^{n} w_{I} d \xi_{i} \simeq \int_{\xi_{a}, \tau_{b}, \xi_{c}} \sum_{a, b, c} \cot \left(\theta_{i}\right) d \xi_{i}
$$

\subsection{Discrete Energy via Weighted Barycentric Coordinates}

We will now prove that the energy expression of (46) can be used to determine the energy at each element. To that end, we here restrict to two-dimensional case, and thus we first prove that the integrant of (46), which can be identified as the differential form

$$
\omega=\sum_{a, b, c} \cot \left(\theta_{i}\right) d \xi_{i}=\cot \left(\theta_{a}\right) d \xi_{a}+\cot \left(\theta_{b}\right) d \xi_{b}+\cot \left(\theta_{c}\right) d \xi_{c}
$$

is a closed 1-form, see in [12-14]. The proof can be based on observing that $d \omega$ can be written as

$$
\begin{aligned}
d \omega= & {\left[\frac{\partial \cot \left(\theta_{b}\right)}{\partial \xi_{a}}-\frac{\partial \cot \left(\theta_{a}\right)}{\partial \xi_{b}}\right] d \xi_{a} \wedge d \xi_{b} } \\
& +\left[\frac{\partial \cot \left(\theta_{c}\right)}{\partial \xi_{b}}-\frac{\partial \cot \left(\theta_{b}\right)}{\partial \xi_{c}}\right] d \xi_{b} \wedge d \xi_{c} \\
& +\left[\frac{\partial \cot \left(\theta_{a}\right)}{\partial \xi_{c}}-\frac{\partial \cot \left(\theta_{c}\right)}{\partial \xi_{a}}\right] d \xi_{c} \wedge d \xi_{a} .
\end{aligned}
$$

To show that $d \omega=0$ is then straightforward using (45).

Finally, the differential form defined in (46) is exact and thus the integration is strictly path-dependent, and thus completely defines an energy functional for the system.

\section{Summary and Conclusions}

In this work, a geometric formulation of nonlinear elasticity, based on discrete energy functional, is presented. In the first step, discrete energy of tetrahedral elements has been formulated through a map between initial and current positions of their vertices and the use of standard barycentric coordinates. The energy functional of the resulted boundary 
value problem has been minimised using energy consistent technique for constrained systems, where the constraints are enforced by Lagrange multipliers and are eliminated via pre-multiplication of the discrete equations of motion by a discrete null-space matrix of the constraint gradient. Although not tested specifically here, the convergence of the proposed scheme should inherit the convergence of the well-known methods for solving constrained systems by utilising the discrete null-space method. The numerical examples presented - cantilever beam and cube with a spherical hole-demonstrate the capabilities of the approach.

Because the use of standard barycentric coordinates is restricted to three-dimensional convex polytopes, a natural extension of the existing definitions to discrete domains consisting of arbitrary polytopes has been proposed. This opens the possibility to analyse domains with arbitrary cell shapes, including non-convex cells that posses specific microstructural features. The proposed technique is presently suited to conservative problems, i.e., linear and nonlinear elasticity, but it can be extended to dissipative systems, provided that the dissipation mechanism is given in terms of the deformations and stresses calculated in this work. Such extensions are the subject of future work.

Author Contributions: Conceptualization, O.K.; methodology, O.K. and A.P.J.; investigation, O.K., P.B. and A.P.J.; writing — original draft preparation, O.K. and A.P.J.; writing-review \& editing, O.K., P.B and A.P.J.; visualization, O.K. and P.B. All authors have read and agreed to the published version of the manuscript.

Funding: This research was funded by Engineering and Physical Sciences Research Council (EPSRC) UK grant number EP/N026136/1.

Acknowledgments: Authors wish to acknowledge the support of Engineering and Physical Sciences Research Council (EPSRC) UK via grand EP/N026136/1 "Geometric Mechanics of Solids".

Conflicts of Interest: The authors declare no conflict of interest.

\section{Appendix A. Principle of Stationary Action in Elasticity}

In order to derive the equations that describe the deformation of the material we consider its energy per unit volume $\xi_{i}$ as a function of the points on the deformed configuration $x_{i}$ and the relative displacements of neighbouring points $\frac{\partial x_{i}}{\partial X_{j}}$, i.e.,

$$
\kappa_{i} \equiv \kappa_{i}\left(x_{i}, \frac{\partial x_{i}}{\partial X_{j}}\right)
$$

for more details see [3]. The total energy of the body can be then calculated as the volume integral

$$
\mathcal{H}=\int \kappa_{i} d V
$$

or

$$
\mathcal{H}=\iiint \kappa_{i}\left(x_{i}, \frac{\partial x_{i}}{\partial X_{j}}\right) d X_{1} d X_{2} d X_{3}, \quad i, j=1,2,3 .
$$

The principle of stationary action states that this functional must be stationary for the true deformation of the body, i.e., $\delta \mathcal{H}=0$, which can be interpreted as body points under deformation move so as to minimise this energy. Calculating the variation of the energy functional leads to the Euler-Lagrange equations [1]

$$
\frac{d}{d X_{j}} \frac{\partial \mathcal{H}}{\partial\left(\partial x_{i} / \partial X_{j}\right)}-\frac{\partial \mathcal{H}}{\partial x_{i}}=0, \quad i, j=1,2,3
$$

The later three equations are the differential equations of elasticity, applicable to both infinitesimal and finite deformations. 
The total energy (A3) can be represented as a sum of an energy associated with deformation $\mathcal{H}^{D}$ and an energy associated with body forces $\mathcal{H}^{F}$, where the former is a function of relative positions only

$$
\mathcal{H}^{D} \equiv \mathcal{H}^{D}\left(\frac{\partial x_{i}}{\partial X_{j}}\right),
$$

and the latter is a function of absolute positions only $[1,4]$

$$
\mathcal{H}^{F} \equiv \mathcal{H}^{F}\left(x_{i}\right) .
$$

Importantly, $\mathcal{H}^{\mathrm{D}}$ must be invariant of coordinate translations and rotations, leading to a requirement for the deformation energy to be a function of some combination of the principal invariants of the gradient of $\varphi$ defined by (3), see also [1-4].

\section{Appendix B. Boundary Conditions}

The boundary conditions for Equation (A4) are Dirichlet for prescribed (known) displacements and Neumann for prescribed (known) forces/ tractions. The application of the Dirichlet boundary conditions is straightforward in our formulation as the primal unknowns are the nodal displacements. For the Neumann boundary conditions we derive the following.

The equilibrium of the entire domain is expressed by $[1-3,6]$

$$
\mathbb{F}=\int_{\Omega} f d V=-\int_{\partial \Omega} t d S=\mathbb{T}
$$

which shows that that the resultant body force $\mathbb{F}$ must be equal and opposite to the resultant of the applied forces on the boundary.

With the energy definition of Section 2 for a deformation of a body, the internal forces can be defined as the negative gradient of the energy, i.e.,

$$
\mathbb{F}=-\nabla_{\mathbb{X}} \mathcal{H}
$$

or

$$
F_{i}=-\iiint \frac{\partial \mathcal{H}}{\partial x_{i}} d X_{1} d X_{2} d X_{3}
$$

which, when using (A4) becomes

$$
F_{i}=-\iiint \frac{d}{\partial X_{j}} \frac{\partial \mathcal{H}}{\partial\left(\partial x_{i} / \partial X_{j}\right)} d X_{1} d X_{2} d X_{3}
$$

If we further apply the divergence theorem we have and expression of the $i$-th component of the force

$$
F_{i}=-\int \frac{\partial \mathcal{H}}{\partial\left(\partial x_{i} / \partial X_{j}\right)} d A_{j}
$$

at a surface defined its area vector $d A_{j}$. The balance of these forces with the surface forces as in (A7) leads to an expression for the the surface forces that uses the definition of the energy per unit volume, i.e.,

$$
d T_{i}=\frac{\partial \mathcal{H}}{\partial\left(\partial x_{i} / \partial X_{j}\right)} d A_{j}
$$

This expression for the Neumann boundary conditions is used in the current work (see also [6]). 


\section{Appendix C. Navier-Cauchy Equations of Linear Elasticity}

Following the work in [6], we consider the displacement field $u=\mathbb{x}-\mathbb{X}$ and define the symmetric part of its gradient by

$$
u_{i k}=\frac{1}{2}\left(\frac{\partial u_{i}}{\partial X_{k}}+\frac{\partial u_{k}}{\partial X_{i}}\right)
$$

The energy of (A3) can be considered as

$$
\mathcal{H}=\mathcal{H}^{d}=\left.\mathcal{H}^{d}\right|_{0}+\frac{\lambda}{2} u_{i i}^{2}+\mu u_{i k}^{2}
$$

where $\left.\mathcal{H}^{d}\right|_{0}$ corresponds to no change of energy state, thus no deformation. The parameters $\lambda$ and $\mu$ are the Lamé parameters that can be calculated as [6]:

$$
\begin{aligned}
\lambda= & 4\left(\left.\frac{\partial^{2} \mathcal{H}^{d}}{\partial \mathbb{I}_{1} \partial \mathbb{I}_{1}}\right|_{0}\right)+16\left(\left.\frac{\partial^{2} \mathcal{H}^{d}}{\partial \mathbb{I}_{2} \partial \mathbb{I}_{1}}\right|_{0}\right)+4\left(\left.\frac{\partial^{2} \mathcal{H}^{d}}{\partial \mathbb{I}_{3} \partial \mathbb{I}_{1}}\right|_{0}\right)+16\left(\left.\frac{\partial^{2} \mathcal{H}^{d}}{\partial \mathbb{I}_{2} \partial \mathbb{I}_{2}}\right|_{0}\right) \\
& +8\left(\left.\frac{\partial^{2} \mathcal{H}^{d}}{\partial \mathbb{I}_{3} \partial \mathbb{I}_{2}}\right|_{0}\right)+\left(\left.\frac{\partial^{2} \mathcal{H}^{d}}{\partial \mathbb{I}_{3} \partial \mathbb{I}_{3}}\right|_{0}\right)-2 \frac{\partial \mathcal{H}^{d}}{\partial \mathbb{I}_{1}}
\end{aligned}
$$

and

$$
\mu=\left.2\left(\frac{\partial \mathcal{H}^{d}}{\partial \mathbb{I}_{1}}+\frac{\partial \mathcal{H}^{d}}{\partial \mathbb{I}_{2}}\right)\right|_{0}
$$

Using the energy of (A14) in the Euler-Lagrange Equation (A4), together with the definitions of the Lamé constants (A15) and (A16) we get the equilibrium equations for linear isotropic elastic materials (with no gravitational body force)

$$
\mu \frac{\partial^{2} u_{i}}{\partial\left(X_{k}\right)^{2}}+(\lambda+\mu) \frac{\partial^{2} u_{l}}{\partial X_{i} \partial X_{l}}=0
$$

which can be written in terms of Youngs modulus

$$
E=\frac{\mu(3 \lambda+2 \mu)}{\lambda+\mu}
$$

and Poisson's ratio

$$
v=\frac{\lambda}{2(\lambda+\mu)}
$$

as

$$
\frac{E}{2(1+v)} \frac{\partial^{2} u_{i}}{\partial\left(X_{k}\right)^{2}}+\frac{E}{2(1+v)(1-2 v)} \frac{\partial^{2} u_{l}}{\partial X_{i} \partial X_{l}}=0 .
$$

Equations (A17) and (A20) are the Navier-Cauchy equilibrium equations for linear elasticity.

\section{References}

1. Marsden, J.; Hughes, T. Mathematical Foundations of Elasticity; Dover Civil and Mechanical Engineering Series; Courier Corporation: Dover, UK, 1994.

2. Gurtin, M.E. The Linear Theory of Elasticity. In Linear Theories of Elasticity and Thermoelasticity: Linear and Nonlinear Theories of Rods, Plates, and Shells; Truesdell, C., Ed.; Springer: Berlin/Heidelberg, Germany, 1973; pp. 1-295.

3. Spencer, A. Continuum Mechanics; Dover Books on Physics; Dover Publications: Dover, UK, 2012.

4. Steinmann, P. Geometrical Foundations of Continuum Mechanics: An Application to First- and Second-Order Elasticity and ElastoPlasticity; Lecture Notes in Applied Mathematics and Mechanics; Springer: Berlin/Heidelberg, Germany, 2015.

5. Shabana, A.A. Computational Continuum Mechanics; Cambridge University Press: Cambridge, UK, 2008. 
6. Landau, L.; Pitaevskii, L.; Kosevich, A.; Lifshitz, E. Theory of Elasticity: Volume 7; Number $\tau$. 7; Elsevier Science: Amsterdam, The Netherlands, 2012.

7. Rashid, M.M.; Selimotic, M. A three-dimensional finite element method with arbitrary polyhedral elements. Int. J. Numer. Methods Eng. 2006, 67, 226-252. [CrossRef]

8. Sohn, D.; Cho, Y.S.; Im, S. A novel scheme to generate meshes with hexahedral elements and poly-pyramid elements: The carving technique. Comput. Methods Appl. Mech. Eng. 2012, 201-204, 208-227. [CrossRef]

9. Sohn, D.; Han, J.; Cho, Y.S.; Im, S. A finite element scheme with the aid of a new carving technique combined with smoothed integration. Comput. Methods Appl. Mech. Eng. 2013, 254, 42-60. [CrossRef]

10. Beirão Da Veiga, L.; Brezzi, F.; Cangiani, A.; Manzini, G.; Marini, L.D.; Russo, A. Basic principles of Virtual Element Methods. Math. Model. Methods Appl. Sci. 2013, 23, 199-214. [CrossRef]

11. Gain, A.L.; Talischi, C.; Paulino, G.H. On the Virtual Element Method for three-dimensional linear elasticity problems on arbitrary polyhedral meshes. Comput. Methods Appl. Mech. Eng. 2014, 282, 132-160. [CrossRef]

12. Desbrun, M.; Hirani, A.N.; Leok, M.; Marsden, J.E. Discrete Exterior Calculus. arXiv 2005, arXiv:math/0508341.

13. Gillette, A.; Bajaj, C. Dual formulations of mixed finite element methods with applications. CAD Comput. Aided Des. 2011, 43, 1213-1221. [CrossRef] [PubMed]

14. Kosmas, O.; Jivkov, A. Development of geometric formulation of elasticity. In Proceedings of the 1st International Conference on Theoretical, Applied, Experimental Mechanics, Cyprus, Greece, 17-20 June 2018; pp. 262-267.

15. Dassios, I.; Jivkov, A.; O'Ceeffe, G. A mathematical model for elasticity using calculus on discrete manifolds. Math. Methods Appl. Sci. 2018, 41, 9057-9070. [CrossRef]

16. Seruga, D.; Kosmas, O.; Jivkov, A. Geometric modelling of elastic and elastoplastic solids with separation of volumetric and distortional energies and Prandtl operators. Int. J. Solids Struct. 2020, 198, 136-148. [CrossRef]

17. Dassios, I.; Jivkov, A.; Abu-Muharib, A.; James, P. A mathematical model for plasticity and damage: A discrete calculus formulation. J. Comput. Appl. Math. 2017, 312, 27-38. [CrossRef]

18. Tsukerman, I. Spurious numerical solutions in electromagnetic resonance problems. IEEE Trans. Magn. 2003, 39, 1405-1408. [CrossRef]

19. Millán, D.; Sukumar, N.; Arroyo, M. Cell-based maximum-entropy approximants. Comput. Methods Appl. Mech. Eng. 2015, 284, 712-731. [CrossRef]

20. Bassi, F.; Botti, L.; Colombo, A.; Pietro, D.D.; Tesini, P. On the flexibility of agglomeration based physical space discontinuous Galerkin discretizations. J. Comput. Phys. 2012, 231, 45-65. [CrossRef]

21. Sansour, C. On the geometric structure of the stress and strain tensors, dual variables and objective rates in continuum mechanics. Arch. Mech. 1992, 44, 527-556.

22. Svendsen, B. A local frame formulation of dual-strain pairs and time derivatives. Arch. Mech. 1995, 111, 13-40. [CrossRef]

23. van der Giessen, E.; Kollmann, F.G. On Mathematical Aspects of Dual Variables in Continuum Mechanics. Part 1: Mathematical Principles. ZAMM J. Appl. Math. Mech. Z. Angew. Math. Mech. 1996, 76, 447-462. [CrossRef]

24. Stumpf, H.; Hoppe, U. The Application of Tensor Algebra on Manifolds to Nonlinear Continuum Mechanics: Invited Survey Article. ZAMM J. Appl. Math. Mech. Z. Angew. Math. Mech. 1997, 77, 327-339. [CrossRef]

25. Kadianakis, N. On the Geometry of Lagrangian and Eulerian Descriptions in Continuum Mechanics. ZAMM J. Appl. Math. Mech. Z. Angew. Math. Mech. 1999, 79, 131-138. [CrossRef]

26. Betsch, P.; Leyendecker, S. The discrete null space method for the energy consistent integration of constrained mechanical systems. Part II: multibody dynamics. Int. J. Numer. Methods Eng. 2006, 67, 499-552. [CrossRef]

27. Betsch, P. The discrete null space method for the energy consistent integration of constrained mechanical systems: Part I: Holonomic constraints. Comput. Methods Appl. Mech. Eng. 2005, 194, 5159-5190. [CrossRef]

28. Kosmas, O.; Papadopoulos, D.; Vlachos, D. Geometric Derivation and Analysis of Multi-Symplectic Numerical Schemes for Differential Equations. Comput. Math. Var. Anal. 2020, 159, 207-226.

29. Kosmas, O.; Leyendecker, S. Family of higher order exponential variational integrators for split potential systems. J. Phys. Conf. Ser. 2015, 574, 012002. [CrossRef]

30. Kosmas, O.; Vlachos, D. A space-time geodesic approach for phase fitted variational integrators. J. Phys. Conf. Ser. 2016, 738, 012133. [CrossRef]

31. Leitz, T.; Leyendecker, S. Galerkin Lie-group variational integrators based on unit quaternion interpolation. Comput. Methods Appl. Mech. Eng. 2018, 338, 333-361. [CrossRef]

32. Chati, M.K.; Mukherjee, S.; Paulino, G.H. The meshless hypersingular boundary node method for three-dimensional potential theory and linear elasticity problems. Eng. Anal. Bound. Elem. 2001, 25, 639-653. [CrossRef]

33. Floater, M.S.; Hormann, K.; Kós, G. A general construction of barycentric coordinates over convex polygons. Adv. Comput. Math. 2006, 24, 311-331. [CrossRef] 